\section{Ball lightning}

SIR - Singer in News and Views recently discussed ${ }^{1}$ ideas about the origin of ball lightning in the context of Ohtsuki and Ofuruton's report ${ }^{2}$ on fireballs generated in air subjected to microwaves. Singer was wrong to assert that I have concluded that ball lightning is not a plasmoid structure. Quite the contrary. I believe the failure of most plasmoid models is attributable to their use of an invalid single-fluid plasma approximation of the virial theorem. Details of my alternative two-fluid plasmoid model are reported in ref. 3 .

\section{National Defence Research \\ Establishment,}

ERIK A. WITALIS

S-172 90 Sundbyberg, Sweden

1. Singer, S. Nature 350, 108-109 (1991).

2. Ohtsuki, Y. H. \& Ofuruton, H. Nature 350, 139-141 (1991).

3. Witalis, E. A. J. Meteorol. 15, 121-128 (1990),

\section{Plastid origins}

SIR - We are concerned over the increasingly confused (and confusing) use of the terms monophyletic and polyphyletic in connection with the origin of plastids and mitochondria. The News and Views ${ }^{1}$ that accompanied our recent paper $^{2}$ refers to the fact that we show that plastids have polyphyletic origins. Raven ${ }^{3}$ uses the term polyphyly to mean that plastids in different groups of plants and algae arose, through endosymbiosis, from different prokaryotes with different characteristics.

By comparing eukaryotic-type small subunit ribosomal RNA sequences from a cryptomonad alga, we provide evidence supporting the view that more than one endosymbiotic event has contributed to the diversity of plastids seen today. But we do not interpret our results to mean that plastids have polyphyletic origins. It is quite possible that plastids, having arisen several times as a result of secondary endosymbiotic events, nevertheless had only a single primary (monophyletic) origin ${ }^{4}$. Further comparisons of genes encoded in plastid genomes are needed to decide this issue ${ }^{5}$.

Susan E. Douglas

Institute of Marine Biosciences,

National Research Council of Canada,

Halifax, Nova Scotia B3H 3Z1, Canada

Department of Biochemistry,

Michael W. Gray

Dalhousie University,

Halifax, Nova Scotia B3H 4H7, Canada

1. Penny, D. \& O'Kelly, C. Nature 350, 106-107 (1991)

2. Douglas, S. E et al. Nature 350, 148-151 (1991)

3. Raven, P. H. Science 169, 641-646 (1970)

4. Cavalier-Smith, T. Biol. J. Linn. Soc. 17, 289 (1982)

5. Gray, M. W. in The Molecular Biology of Plastids (eds Bogorad, L. \& Vasil, I. K.) 303-330 (Academic, San Diego, 1991).

\title{
Similarities in RNA helicases
}

SIR - There is much excitement about the (putative) RNA helicases from various organisms involved in pre-messenger RNA splicing, ribosomal RNA maturation, translation initiation, ribosome assembly and probably also in other processes related to cell growth and division. A rapidly expanding number of these enzymes, for which the amino-acid sequences have been reported, can be assigned to the previously defined superfamily II, which includes as a subdivision the well-known DEAD family and the putative RNA helicases of several groups of positive-strand RNA viruses. In their discussion ${ }^{1}$ of possible functional analogies between the helicases in different systems, Wassarman and Steitz neglected the viral proteins because of the paucity of the evidence relating to their proposed function.

Recently, the RNA helicase and RNA-dependent ATPase activities of one of the proposed RNA viral heli-

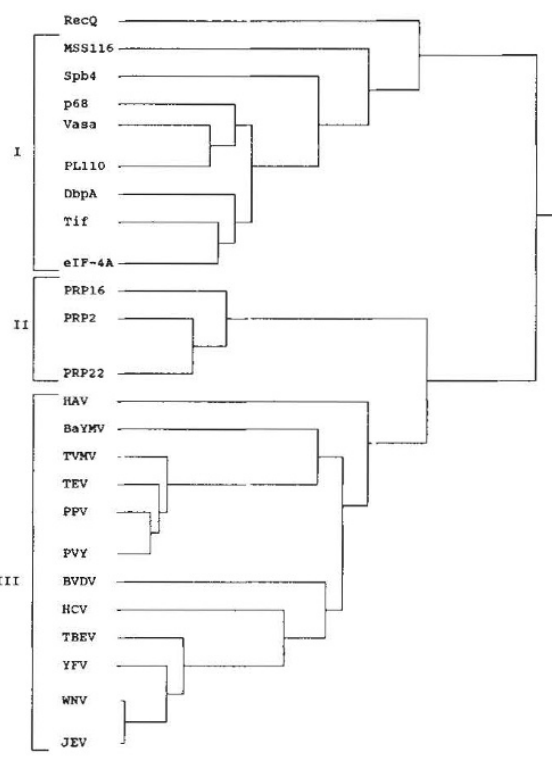

The cluster dendrogram for the cellular and viral helicases was constructed by the UPGMA method ${ }^{6}$. Pairwise distances between the amino-acid sequences were calculated using the method in ref. 7. (Further details of the calculation and the complete list of references are available from the author on request). The groups of proteins are designated: I, DEAD helicases; II DEAH helicases involved in yeast pre-mRNA splicing; III, RNA viral DEAH helicases, RecQ is an Escherichia coli helicase closely related to the DEAD family. BaYMV, barley yellow mosaic virus; TVMV, tobacco vein-mottling virus, PVY, potato virus Y; PPV, plum pox virus; TEV, tobacco etch virus (potyviruses); HCV, hepatitis C virus; TBEV, tick-borne encephalitis virus; YFV, yellow fever virus; WNV, West Nile virus; JEV, Japanese encephalitis virus (flaviviruses); BVDV, bovine viral diarrhoea virus (pestivirus); HAV, Cryphonectria parasitica hypovirulence-associated double-stranded RNA virus. cases, the potyvirus CI protein, have been demonstrated ${ }^{2,3}$. RNA viral helicases and three helicases involved in different steps of yeast pre-mRNA splicing, PRP2, PRP16 and PRP22, share two sequence signatures, DExH and QxxGRxxR, in the conserved motifs II and VI (ref. 4), as opposed to DEAD and HxxGRxxR in the proteins of the DEAD family ${ }^{5}$.

I have generated a sequence alignment of the entire set of RNA viral helicase belonging to superfamily II, the PRP proteins and those of the DEAD family for the region spanning all seven conserved motifs, and have derived a cluster dendrogram (see figure). The resulting branching order is clear-cut in showing the association between the RNA viral helicases and the PRP proteins.

Whatever the evolutionary bearing of these observations, they indicate that the sequences of the helicases mediating certain steps of pre-mRNA splicing are specifically related to those involved in viral RNA replication. Thus, mechanistic parallels in the helicase action could be even more profound between viral RNA replication and splicing than between splicing and translation, as suggested by Wassarman and Steitz ${ }^{1}$. These authors discussed the possibility that the helicases of this superfamily might ensure the fidelity required for splicing and translation.

Following this line of reasoning, the helicases of RNA viruses may also reduce the error rate of RNA replication in a manner analogous to that exploited during splicing. This might help to rationalize our previous observation $^{6}$ that relatively large positivestrand RNA viral genomes (greater than five kilobases) all encode the putative RNA helicase, whereas smaller viral genomes lack the respective gene. By reducing the nucleotide misincorporation rate, the helicases may ensure the very possibility of the existence of the larger viral genomes.

Department of Biology,

EUGENE V. KOONIN

Texas A\&M University,

College Station,

Texas 77843-3258, USA

Permanent address: Institute of Microbiology. Academy of Sciences, 7 Prosp. 60let Oktyabrya, 117811 Moscow. USSR.

1. Wassarman, D. A. \& Steitz, J. A. Nature 349, 463-464 (1991).

. Lain, S., Riechmann, J. \& Garcia, J. A. Nucleic Acids Res. 18, 7003-7006 (1990).

3. Lain, S. et al. J. Virol. 65, 1-6 (1991)

4. Gorbalenya, A. E. et. al. Nucleic Acids Res. 17, 47134730 (1989).

5. Company, M., Arenas, J. \& Abelson, J. Nature 349 487-493 (1991)

6. Gorbalenya, A. E. \& Koonin, E. V. Nucleic Acids Res. 17 8413-8440 (1989)

7. Feng, D. F., Johnson, M. S. \& Doolittle, R. F. J. molec. Evol. 21, 112-125 (1985). 